\title{
Left sided valvar regurgitation in normal children and adolescents
}

\author{
J D R Thomson, J Allen, J L Gibbs
}

\begin{abstract}
Objective-To determine the prevalence and characteristics of left sided valvar regurgitation in normal children and adolescents.

Design-Prospective observational study.

Setting-Tertiary paediatric referral centre.

Patients-329 volunteers (194 male, 135 female, age range 3-18 years).

Main outcome measures-Detection of regurgitation with colour flow mapping after valve closure. Measurement of jet area, maximal velocity, and duration.

Results-Mitral regurgitation was present in six subjects $(1.82 \%, 95 \%$ confidence interval (CI) $0.38 \%$ to $3.3 \%$ ) and was not seen before 7 years of age. The jets ranged from 1.1 to $1.9 \mathrm{~cm}^{2}$ (mean $1.4 \mathrm{~cm}^{2}$ ) in area and were confined to the proximal half of the left atrium. All of the detectable jets were pansystolic and five of six arose from the posteriomedial aspect of the mitral valve. Aortic regurgitation was seen in one girl aged 11 years $(0.3 \%, 95 \%$ CI $0 \%$ to $0.9 \%)$. The signal was pandiastolic and $0.44 \mathrm{~cm}^{2}$ in area.

Conclusions-True mitral regurgitation occurring after rather than during mitral valve closure was detected in $<2 \%$ of subjects. These data support previous work in adult patients suggesting that trivial degrees of mitral regurgitation may be related to the process of aging. Aortic regurgitation is very rare in normal children and adolescents and should not be considered as a normal finding.

(Heart 2000;83:185-187)
\end{abstract}

Keywords: left sided valvar regurgitation; paediatric cardiology

Clinically silent, very mild degrees of left heart valve regurgitation have been reported to occur in a proportion of the normal population. ${ }^{1-15}$ Most of the data regarding prevalence of echocardiographically detected valvar regurgitation in normal subjects have related to adults $^{1-6}$ and some studies have suggested increasing prevalence with age. ${ }^{347}$ To determine the prevalence and characteristics of left sided regurgitation in normal children and adolescents, Doppler echocardiography was used to study prospectively 329 normal volunteers aged between 3 and 18 years.

\section{Patients and methods}

Informed consent was sought from healthy volunteers from three local schools. Subjects were screened for illness (both cardiac and non-cardiac) by questionnaire, and children with any past medical history were excluded from the study. The study population comprised 329 subjects, 194 male and 135 female (age range 3-18 years, median 11 years). Approval for the study was granted by the ethics committee of the United Leeds Teaching Hospitals NHS trust.

Ultrasound examinations were performed using a Hewlett Packard Sonos 1500 phased array imaging system (Hewlett Packard, Andover, Massachusetts, USA). Real time images were recorded on high quality $3 / 4$ inch super VHS video tape using a Panasonic AG-7340 video recorder. Transducers with frequencies ranging from $2.0-7.5 \mathrm{MHz}$ were required because of the age range of the study population. In each subject the highest fre- quency of transducer giving adequate penetration was used. Flow patterns across the valves were assessed using pulsed, continuous, and colour flow Doppler techniques. For colour flow mapping the wall filter was set at $0.21 \mathrm{~m} / \mathrm{s}$ and the frame rate at $12-14$ frames/s. A variance map was used so that high velocity blood flow was displayed as a mosaic pattern. Colour Doppler was optimised by the standard technique; the colour gain settings were turned down completely and gradually increased until static background noise barely appeared. Spectral analysis using pulsed Doppler was obtained with a sample volume of $2.5 \mathrm{~mm}$.

Examinations were carried out with the subjects in the lateral decubitus position. A single lead ECG was monitored simultaneously for timing of the phases of the cardiac cycle. Cross sectional imaging and colour flow Doppler was performed to assess the mitral and aortic valves from the standard parasternal long and short axis, four chamber, and ventricular short axis views. Mitral forward flow velocity was assessed using pulsed wave Doppler. Aortic forward flow was assessed using a dedicated continuous wave $1.9 \mathrm{MHz}$ probe from the suprasternal notch. Valvar regurgitation was said to be present when high velocity colour flow, directed into the proximal chamber, was present after valve closure. If valve regurgitation was detected by colour flow techniques continuous wave Doppler and colour $M$ mode were employed to characterise the jet further (figs 1 and 2). Signals of very short duration detected during valve closure and localised to the region of valve coaptation were not 

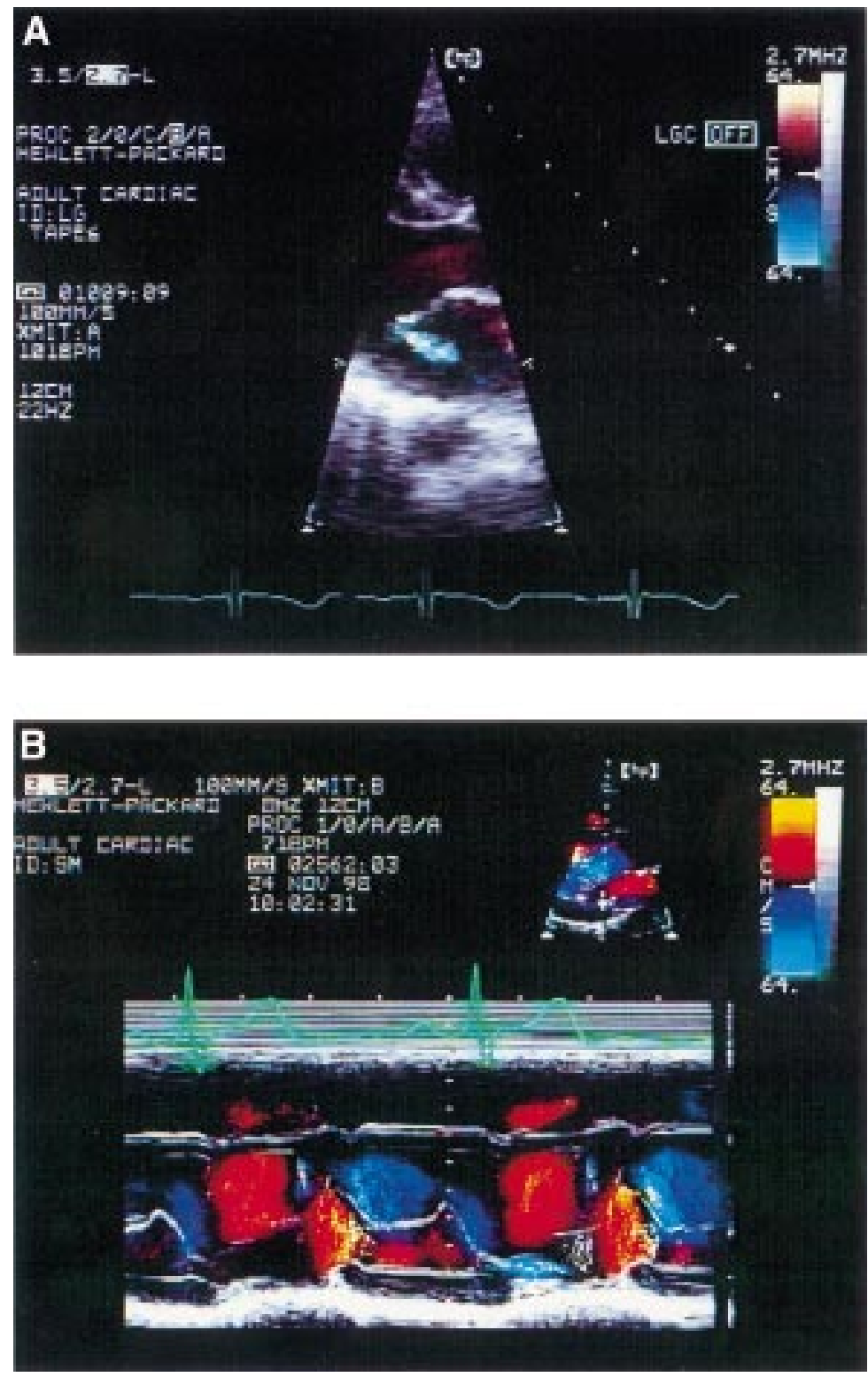

Figure 1 Cross sectional, parasternal long axis view (A) and colour $M$ mode (B) recordings, showing a jet of "true" mitral regurgitation occurring after valve leaflet closure (arrowed). regurgitant jet was pansystolic (fig 1). Because of the weak signal associated with trivial degrees of regurgitation it was not possible to get a spectral waveform with a sufficiently clear peak to allow for accurate quantification of the maximal velocity of the regurgitant jet. However, in all cases the jet clearly exceeded $3 \mathrm{~m} / \mathrm{s}$.

Aortic regurgitation was detected in one female subject aged 11 years $(0.3 \%, 95 \%$ CI $0 \%$ to $0.9 \%$ ). The jet was central and measured $0.44 \mathrm{~cm}^{2}$. The signal was pandiastolic with a poorly defined but high velocity spectral envelope.

\section{Discussion}

While it is generally accepted that trivial right sided regurgitation is a common, benign finding in normal subjects at any age, the significance of left sided regurgitation is less clear. There has been debate about both the significance of trivial left sided valve regurgitation in hearts that appear otherwise normal and the mechanisms underlying it. ${ }^{3689}$ In particular the need for antibiotic prophylaxis has been debated, with the possibility that even trivial valve regurgitation may predispose to bacterial endocarditis. ${ }^{8}$ Previous studies have been almost entirely in adults. ${ }^{1-6}$ In two studies ${ }^{36}$ small numbers of children were included without detailed age stratification. The one report in which 461 children were specifically considered was retrospective and related to children referred for specialist cardiac opinion because they were suspected of having cardiovascular disease.${ }^{10}$ Studies in normal adults suggest that valve regurgitation is relatively common with advancing age. In some series mitral regurgitation has been reported in $100 \%$ of subjects. ${ }^{11}$ However, criteria for diagnosis of regurgitation have varied, ${ }^{3568}$ with some groups accepting short signals occurring during rather than after valve closure as representing regurgitation.

In our study we adhered to a strict definition of mitral regurgitation occurring only after closure of the valve leaflets. In all subjects meeting these criteria the jet was pansystolic (fig 1B). Our results showed that Doppler detectable left sided valve regurgitation in normal children is rare. Mitral regurgitation occurred more commonly than aortic regurgitation. None of the cases with regurgitation in our study had clinical signs of valve incompetence, and in all cases the regurgitation appeared trivial. Colour signals occurring during mitral valve closure were commonly seen (68/322 of our cases); these signals were transient (lasting only for a few milliseconds), never aliased on colour Doppler, and were not detectable with continuous wave Doppler echocardiography. We were able to confirm both the presence and the short duration of these jets using colour $\mathrm{M}$ mode (fig 2). These signals occurring during valve closure were common across the whole age range studied whereas true regurgitation was not seen before 7 years of age. Interestingly small colour signals during valve closure were consistently seen in the same region of the valve as the true regurgitant jets. It is likely these colour signals represent retrograde, but low velocity blood movement as a consequence of valve gitation arose from the posteriomedial aspect of the mitral valve. In all six cases the 

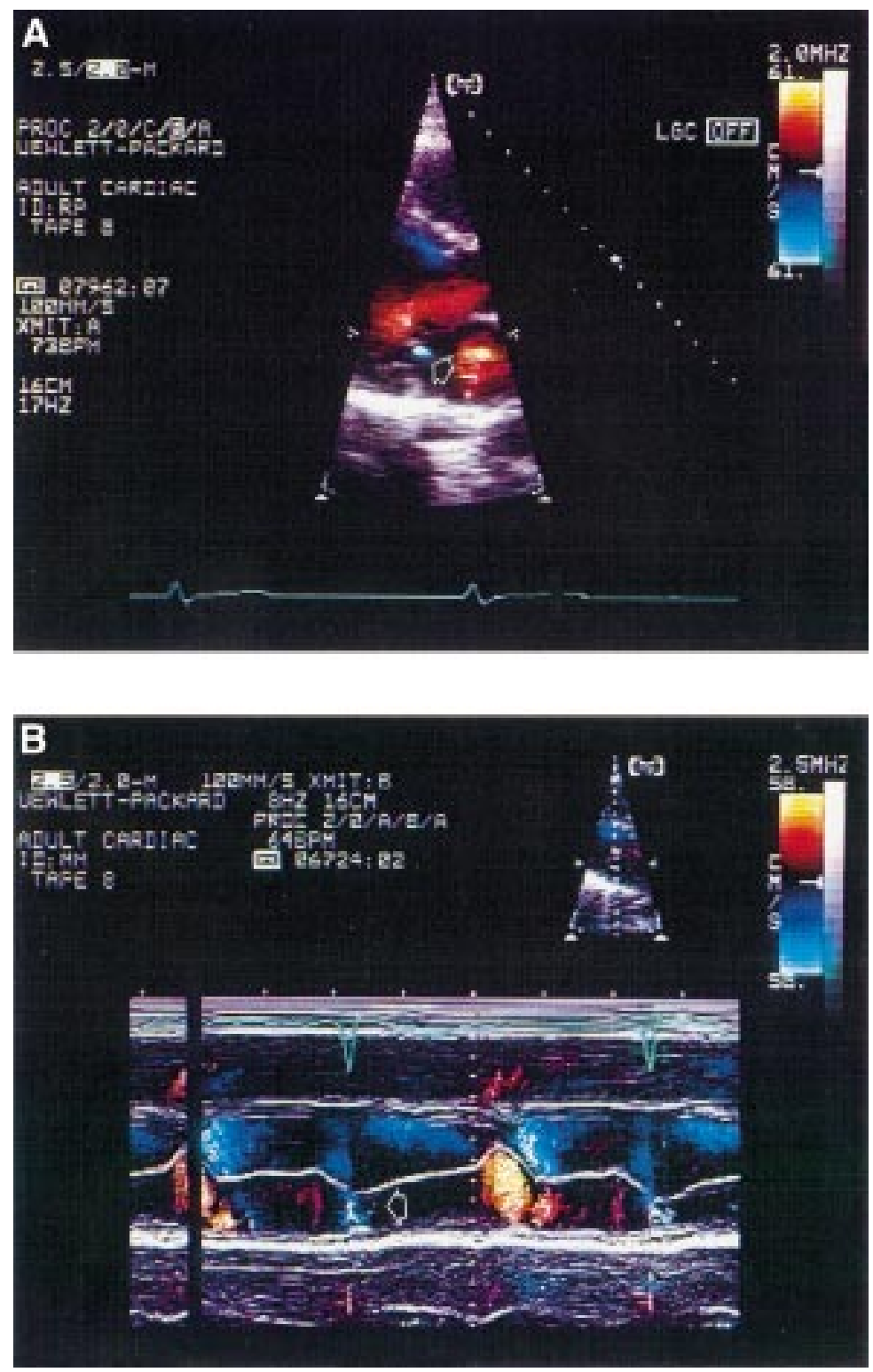

Figure 2 Cross sectional, parasternal long axis view ( $A$ ) and colour $M$ mode (B) showing the transient colour signal commonly seen during valve closure (arrowed).

closure. In our study group we found only one case with trivial aortic incompetence. Aortic regurgitation was not detected in the other study reporting significant numbers of children.
CONCLUSIONS

Aortic regurgitation is very rare $(0.3 \%)$ in normal children and adolescents. Therefore genuine aortic regurgitation should not be regarded as a normal finding in these subjects. Trivial mitral regurgitation occurs in $<2 \%$ of normal children and adolescents. Studies of normal adults suggest that similarly trivial degrees of mitral regurgitation can be detected in up to $100 \% .^{11}$ Our study provides supportive evidence for previous suggestions that trivial degrees of mitral regurgitation are likely to be related to the process of aging. It seems possible that trivial, subclinical valvar regurgitation might be the substrate for the rare but well recognised phenomenon of infective endocarditis in a previously "normal heart".

1 Yock PG, Schnittger I, Popp RL. Is continuous wave Doppler too sensitive in diagnosing pathological valvular
regurgitation? [abstract] Circulation 1984;70(suppl II):381.

2 Berger M, Hecht SR, Van Tosh A, et al. Pulsed and continuous wave Doppler echocardiographic assessment of valvular regurgitation in normal subjects. $\mathcal{F} \mathrm{Am}$ Coll Cardiol 1989;13:1540-5.

3 Choong CY, Abascal VM, Weyman J, et al. Prevalence of valvular regurgitation by Doppler echocardiography in patients with structurally normal hearts by two dimensional echocardiography. Am Heart f 1989;11:636-42.

4 Akasaka T, Yoshikawa J, Yoshida K, et al. Age related valvular regurgitation: a study by pulsed Doppler echocardiography Circulation 1987;76:262-5.

5 Kostuki W, Vandenbossche J, Friate A, et al. Doppler regurgitant flow patterns of normal valves. $\mathrm{Am} \mathcal{F}$ Cardiol 1987;76:262-5.

6 Yoshida K, Yoshikawa J, Shakudo, et al. Color Doppler evaluation of valvular regurgitation in normal subjects. Circulation 1988;78:840-7.

7 Klein AL, Burstow DL, Jamil Tajik A, et al. Age related prevalence of valvular regurgitation in normal subjects: a comprehensive color flow examination of 118 volunteers. $\mathcal{F}$ Am Soc Echocardiogr 1990;3:54-63.

8 Houston A. Doppler ultrasound and the apparently normal heart. Br Heart f 1993;69:99-100.

9 Jobic J, Slama M, Tribouiloy C, et al. Doppler echocardiographic evaluation of valve regurgitation in healthy graphic evaluation of valve regurgitat
volunteers. Br Heart $\mathcal{F} 1993 ; 69: 109-13$.

10 Brand A, Dollberg S, Keren A. The prevalence of valvular regurgitation in children with structurally normal hearts: a regurgitation in children with structurally normal hearts: a
color Doppler echocardiographic study. Am Heart f 1992; color Doppler

11 Wittlich N, Sietmer R, Schaudi M, et al. Transesophageal color flow mapping in normal subjects [abstract]. Eur Heart f 1988;9(suppl 1):272

12 Abbasi AS, Allen MW, DeCristofaro D, et al. Detection and estimation of the degree of mitral regurgitation by pulsed Doppler echocardiography. Circulation 1980:61:143-7.

13 Sahn DJ, Maciel BC. Physiological valvular regurgitation and the potential for iatrogenic heart disease. Circulation 1988;78:1075-7.

14 Kostuki W, Vandenbossche JL, Friat A, et al. Pulsed Doppler regurgitant flow patterns of normal valves. Am $\mathcal{f}$ Cardiol 1986;58:309-13

15 Takao S, Miyatake K, Izumi S, et al. Physiological pulmonary regurgitation detected by the Doppler technique and its differential diagnosis [abstract]. $\mathcal{F} \mathrm{Am}$ Coll Cardiol 1985;5:499. 